\title{
Optimal Allocation Problem in the Machine Repairman System with Heterogeneous Servers
}

\author{
Dmitry Efrosinin ${ }^{1, \star}$, Christopher Spannring ${ }^{1}$, and Janos Sztrik ${ }^{2, \star \star}$ \\ 1 Johannes Kepler University Linz, \\ Altenbergerstrasse 69, 4040 Linz, Austria \\ ${ }^{2}$ University of Debrecen, \\ Egyetem ter 1, 4032 Debrecen, Hungary \\ dmitry.efrosinin@jku.at, c.spannring@gmx.at, sztrik.janos@inf.unideb.hu \\ http://www.jku.at, http://www.irh.inf.unideb.hu
}

\begin{abstract}
A controllable repairman model consists of $L$ machines subject to failures and two repair servers working at different speeds. The problem of optimal allocation of failed machines between the servers is examined. The optimal control policy is calculated versus cost structures. As a result the optimal policy can be of threshold type, hysteretic type or have more complicated form. It is shown that the corresponding Markov process for hysteretic control policy belongs to the class of the Quasi-Birth-and-Death processes (QBD) with three diagonal block infinitesimal matrix. The stationary characteristics in this case are derived in matrix analytic form. Some numerical results are used to illustrate a number of features of the controlled model under study.
\end{abstract}

Keywords: Machine repairman system, performance analysis, dynamicprogramming, optimal allocation, threshold policy, hysteretic policy.

\section{Introduction}

The machine repairman systems are normally described by means of the closed queueing systems, i.e. the systems with finite population. In such a system the customers of the finite population are the machines which are working at the operation area and during operation time they can fail independently of each other. The failed machines are sent to the repair facility where they can be restored. After the repair the machine becomes as good as a new one and is returned to the operational area. If all repair servers are busy a just failed machine has to wait for the repair at the buffer. In most cases in multi-server case the repair

\footnotetext{
* This work was funded by the COMET K2 Center "Austrian Center of Competence in Mechatronics (ACCM)", funded by the Austrian federal government, the federal state Upper Austria, and the scientific partners of the ACCM.

** The publication was supported by the TAMOP-4.2.2.C-11/1/KONY-2012-0001 project. The Project has been supported by the European Union, cofinanced by the European Social Fund.
} 
servers are assumed to be homogeneous, i.e. they repair the machines at equal speeds [2. Only few papers deal with heterogeneous servers, see e.g. 9].

The problem of optimal allocation between heterogeneous servers was studied exhaustively only for infinite population queues. In [10] it was shown that the optimal allocation policy in heterogeneous system without preemption and switching costs is of threshold type, i.e. the server with larger mean usage cost has to be used if the queue length reaches some prespecified threshold level. The equivalent system with switching cost was analyzed in [5], where the hysteretic allocation policy took place. Due to this policy the usage of the server with higher mean usage cost is performed via the switch-on and switch-off threshold levels. For some other results concerning the hysteretic policy we refer the reader to 146 .

In this paper we combine the finite population queueing system with heterogeneous repair facility and optimal allocation problem which obviously represents a missing subject among the available results. For the fixed threshold level and specified cost structure we have obtained explicitly the corresponding average cost which was minimized. To calculate the policy we use a dynamicprogramming approach. Several structural properties of a control policy are established as well.

The rest of the paper is organized as follows: Section 2 describes the mathematical model based on a controllable Markov process. In Section 3 optimization problem is formulated and optimal equations for the dynamic-programming value function are derived. Section 4 deals with explicit evaluation of the mean performance measures. Finally, some numerical examples are presented in Section 5 .

\section{Mathematical Model}

Consider the machine repairman system described in introduction. $L$ machines subject to failure are working in- parallel. The operational time of each machine is exponentially distributed with parameter $\lambda$. The machines fail independently of each other. The repair facility consists of two heterogeneous servers with exponential distributed repair times with parameters $\mu_{1}>\mu_{2}>0$. The process of the repair is assumed to be without preemption, i.e. the failed machine can not change the server during the repair process. The operational and repair times are assumed to be mutually independent.

Let $Q(t)$ denote the number of failed machines in the buffer and $D_{i}(t)$ - the state of the $i$ th repair server. The system states at time $t$ are described by a continuous-time Markov process

$$
\{X(t)\}_{t \geq 0}=\left\{Q(t), D_{1}(t), D_{2}(t)\right\}_{t \geq 0}
$$

The controllable model associated with a Markov process $\{X(t)\}_{t \geq 0}$ is a fivetuple

$$
\left\{E, A,\{A(x), x \in E\}, \lambda_{x y}(a), c(x, a)\right\} .
$$


- $E$ is a state space of the process $\{X(t)\}_{t \geq 0}$,

$$
E=\left\{x=\left(q, d_{1}, d_{2}\right) ; q \in\{0,1, \ldots, L\}, d_{j} \in\{0,1\}, q+\sum_{j=1}^{2} d_{j} \leq L\right\} .
$$

Further in the paper the notations $q(x), d_{j}(x), j=1,2$, will be used to specify the certain components of the vector state $x=\left(q, d_{1}, d_{2}\right) \in E$.

- $A=\{0,1,2\}$ is an action space with elements $a \in A$, where $a=j>0$ means "to send a failed machine to the server $j ", j=1,2$, and $a=0$ means "to send a failed machine to the buffer".

- The subsets $A(x) \subseteq A$ of control actions in state $x \in E$, where $A(q, 0,0) \equiv$ $A, A(q, 0,1)=\{0,1\}$ and $A(q, 1,0)=\{0,2\}$.

- $\lambda_{x y}(a)$ is a transition intensity to go from state $x$ to state $y$ under a control action $a$. It is assumed that the model is stable and conservative, i.e.

$$
\begin{aligned}
& \lambda_{x y}(a) \geq 0, y \neq x, \lambda_{x x}(a)=-\lambda_{x}(a)=-\sum_{y \neq x} \lambda_{x y}(a), \lambda_{x}(a)<\infty, \\
& \lambda_{x y}(a)= \begin{cases}\lambda\left[L-q(x)-\sum_{j=1}^{2} d_{j}(x)\right] & y=x+e_{a}, a \in A(x), \\
\mu_{j} d_{j}(x) & y=x-e_{j}, q(x)=0, \\
\mu_{j} d_{j}(x) & y=x-e_{j}-e_{0}+e_{a}, q(x)>0, \\
& a \in A\left(x-e_{j}-e_{0}\right) .\end{cases}
\end{aligned}
$$

The notation $e_{j}$ is used for the vector with 1 in the $j$ th position (beginning from 0 th) and 0 elsewhere.

- $c(x, a)$ is an immediate cost in state $x$ under control action $a$,

$$
\begin{aligned}
& c(x, a)=c(x)+c_{01} \lambda\left[L-q(x)-\sum_{j=1}^{2} d_{j}(x)\right] 1_{\left\{d_{2}(x)=0, a=2\right\}}+ \\
& {\left[c_{01} \mu_{1} d_{1}(x) 1_{\left\{d_{2}(x)=0, a=2\right\}}+c_{10} \mu_{2} d_{2}(x) 1_{\{a=0 \vee a=1\}}\right] 1_{\{q(x)>0\}},} \\
& c(x)=c_{0} q(x)+\sum_{j=1}^{2} c_{j} d_{j}(x)
\end{aligned}
$$

where $c_{0}$ - holding cost per unit of time in the buffer, $c_{j}-$ usage cost of a repair server $j$ per unit of time, $c_{01}$ and $c_{10}$ - fixed costs for switching on and off of the slower repair server. If $c_{0}=c_{j}=1, j=1,2$ and $c_{01}=c_{10}=0$, then $c(x, a)$ represents the number of failed machines in state $x$.

We will next explain how the controller chooses its actions. According to the stationary Markov policy $f: E \rightarrow A$ whenever at a decision epoch the system state is $x \in E$, the controller choses an action $f(x)=a \in A(x) \subseteq A$ regardless of the past history of the system. We have two types of decision epochs:

- just after a failure of a machine at state $x$ the controller chooses an action $a \in A(x)$, which prescribes to allocate the machine to one of available servers or to the buffer; 
- just after a repair completion at server $j$ in state $x$ the controller chooses an action $a \in A\left(x-e_{0}-e_{j}\right)$, which prescribes to take another machine from the queue, if it is not empty, and allocate it to one of available repair servers or put it back to the buffer.

\section{Optimization Problem for Performance Characteristics}

The process $\{X(t)\}_{t \geq 0}$ has a finite state space hence we may guarantee that this process is an irreducible, positive recurrent Markov process defined through its infinitesimal matrix $\Lambda=\left[\lambda_{x y}(f(x))\right]$. As it is known [8], for ergodic Markov process with costs the long-run average cost per unit of time (also referred to as gain) for the policy $f$ coincides with corresponding assemble average,

$$
g^{f}=\lim _{t \rightarrow \infty} \frac{1}{t} V^{f}(x, t)=\sum_{y \in E} c(y, a) \pi_{y}^{f},
$$

where

$$
V^{f}(x, t)=\int_{0}^{t} \sum_{y \in E} \mathbb{P}^{f}[X(u)=y \mid X(0)=x] c(y, a) d u
$$

denotes the total average cost up to time $t$ when the process starts in state $x$ and $\pi_{y}^{f}$ denotes a stationary probability of the process given policy $f$. The policy $f^{*}$ is said to be optimal when for any admissible policy $f$

$$
g^{f^{*}}=\min _{f} g^{f} .
$$

We expect that the gain $g^{f^{*}}$ will be smaller or equal to the gain under other heuristic allocation policies, e.g. Fastest Free Server discipline, which prescribes to use a fastest server among available.

The optimal policy $f^{*}$ can be evaluated by means of a Howard iteration algorithm [3, which constructs a sequence of improved policies until the average cost optimal is reached. The key role in this algorithm is played by the dynamic programming value function $v: E \rightarrow \mathbb{R}_{+}$which indicates a transition effect of an initial state $x$ to the total average cost and satisfies a well-known asymptotic relation,

$$
V^{f}(x, t)=g^{f} t+v^{f}(x)+o(1), x \in E, t \rightarrow \infty .
$$

The functions $V^{f}, v^{f}$ and $g^{f}$ further in the paper will be denoted by $V, v$ and $g$ without upper index $f$.

The system will be uniformized as in Puterman [7] with the uniformization constant

$$
\lambda L+\mu_{1}+\mu_{2}=1,
$$

which can be obtained by time scaling. As it is well known, the optimal policy $f$ and the optimal average cost $g$ are solutions of the optimality equation

$$
B v(x)=v(x)+g,
$$

where $B$ is the dynamic programming operator acting on value function $v$. 
Theorem 1. The dynamic programming operator $B$ is defined as follows

$$
\begin{aligned}
& B v(x)=c(x)+\left[L-q(x)-\sum_{j=1}^{2} d_{j}(x)\right] \lambda \min _{a \in A(x)}\left\{v\left(x+e_{a}\right)+c_{01} 1_{\{a=2\}}\right\}+ \\
& {\left[q(x)+\sum_{j=1}^{2} d_{j}(x)\right] \lambda v(x)+\sum_{j: d_{j}(x)=1} \mu_{j} v\left(x-e_{j}\right) 1_{\{q(x)=0\}}+\sum_{j: d_{j}(x)=0} \mu_{j} v(x)+} \\
& {\left[\mu_{1} d_{1}(x) \min _{a \in A\left(x-e_{1}-e_{0}\right)}\left\{v\left(x-e_{1}-e_{0}+e_{a}\right)+c_{01} 1_{\left\{d_{2}(x)=0, a=2\right\}}\right\}+\right.} \\
& \left.\mu_{2} d_{2}(x) \min _{a \in A\left(x-e_{2}-e_{0}\right)}\left\{v\left(x-e_{2}-e_{0}+e_{a}\right)+c_{10} 1_{\{a=0 \vee a=1\}}\right\}\right] 1_{\{q(x)>0\}} .
\end{aligned}
$$

Proof. The optimality equation is obtained by analyzing the function $V(x, t)$ in some infinitesimal interval $[t, t+d t]$. It leads to the differential equation. Applying further the limit expression

$$
\lim _{d t \rightarrow 0} \frac{V(x, t+d t)-V(x, t)}{d t}=0
$$

and taking into account Markov property of $\{X(t)\}_{t \geq 0}$ with asymptotic relation (44) ones get (6).

Corollary 1. From (6) it follows that the optimal policy $f=\left(f_{0}, f_{1}, f_{2}\right)$ consists of components which specify the control action just after a new arrival in state $x$, just after a service completion at server 1 or 2 for nonempty queue,

$$
\begin{aligned}
& f_{0}(x)=\underset{a \in A(x)}{\arg \min }\left\{v\left(x+e_{0}\right), v\left(x+e_{1}\right) 1_{\left\{d_{1}(x)=0\right\}},\left(v\left(x+e_{2}\right)+c_{01}\right) 1_{\left\{d_{2}(x)=0\right\}}\right\}, \\
& f_{1}(x)=\underset{a \in A\left(x-e_{1}-e_{0}\right)}{\arg \min }\left\{v\left(x-e_{1}\right), v\left(x-e_{0}\right),\left(v\left(x-e_{1}-e_{0}+e_{2}\right)+c_{01}\right) 1_{\left\{d_{2}(x)=0\right\}}\right\}, \\
& f_{2}(x)=\underset{a \in A\left(x-e_{2}-e_{0}\right)}{\arg \min }\left\{v\left(x-e_{2}\right)+c_{10}, v\left(x-e_{2}-e_{0}+e_{1}\right), v\left(x-e_{0}\right)\right\} .
\end{aligned}
$$

In case $c_{01}=c_{10}=0, f_{j}(x)=f_{0}\left(x-e_{j}-e_{0}\right), j=1,2$.

\section{Explicit Evaluation of the Gain Function}

As is shown in Section 5 the optimal control policy can be approximated by the hysteretic policy with two threshold levels $(U, D), D \leq U$, i.e. the slower server must be activated when the queue length reaches the upper bound $U$ and deactivated - when the queue length goes below the lower bound $D$. Under the fixed values $U$ and $D$ the gain $g$ can be evaluated explicitly using the right hand side of (11).

Denote by $\pi$ the row vector of the stationary state probabilities with components $\pi_{x}=\lim _{t \rightarrow \infty} \mathbb{P}[X(t)=x]$. Define the following row-subvectors,

$$
\pi_{00}=\left(\pi_{000}, \pi_{001}\right), \pi_{k}= \begin{cases}\left(\pi_{k 10}, \pi_{k 11}\right) & 0 \leq k \leq U-1 \\ \pi_{k 11} & U \leq k \leq L-2 .\end{cases}
$$

The corresponding transition diagram is shown in Figure 1. 


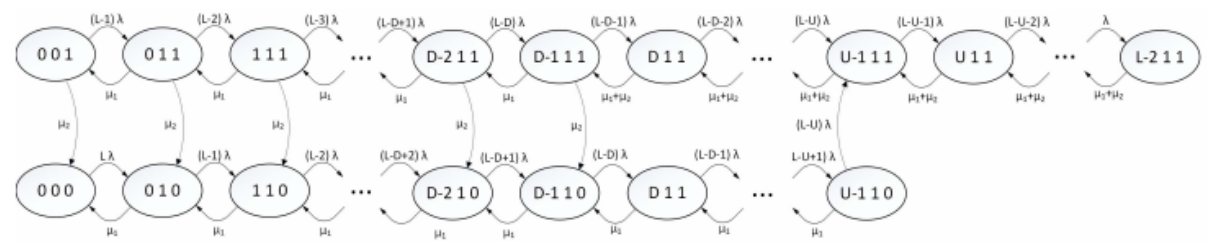

Fig. 1. Transition rate diagram for the hysteretic policy $(U, D)$

Theorem 2. The Markov process $\{X(t)\}_{t \geq 0}$ for the thresholds $(U, D)$ is of the QBD-type (Quasi-Birth-Death) with a state space

$E=\left\{x=\left(q, d_{1}, d_{2}\right) ; d_{j} \in\{0,1\}\right.$ if $q \in\{0,1, \ldots, U-1\}, d_{j}=1$ if $\left.U \leq q \leq L-2\right\}$

and three-diagonal block infinitesimal matrix $\Lambda=\left[\lambda_{x y}(U, D)\right]$ defined as

$\Lambda=\left(\begin{array}{cccccccccccccc}-B_{0} & A_{0}^{0} & 0 & 0 & 0 & 0 & 0 & 0 & 0 & 0 & 0 & 0 & \cdots & 0 \\ C_{0} & -B_{1}^{0} & A_{0}^{1} & 0 & 0 & 0 & 0 & 0 & 0 & 0 & 0 & 0 & \cdots & 0 \\ 0 & \ddots & \ddots & \ddots & \ddots & & & & & \ddots & \ddots & \ddots & \ddots & \vdots \\ 0 & \ldots & C_{0} & -B_{1}^{D-1} & A_{0}^{D} & 0 & 0 & 0 & 0 & 0 & 0 & 0 & \cdots & 0 \\ 0 & \ldots & 0 & C_{1} & -B_{2}^{D} & A_{0}^{D+1} & 0 & 0 & 0 & 0 & 0 & 0 & \cdots & 0 \\ \vdots & \ddots & \ddots & \ddots & \ddots & \ddots & \ddots & \ddots & & \ddots & \ddots & \ddots & \ddots & \vdots \\ 0 & \ldots & 0 & 0 & C_{1} & -B_{2}^{U-2} & A_{0}^{U-1} & 0 & 0 & 0 & 0 & 0 & \cdots & 0 \\ 0 & \ldots & 0 & 0 & 0 & C_{1} & -B_{3} & a_{0}^{U} & 0 & 0 & 0 & 0 & \cdots & 0 \\ 0 & \ldots & 0 & 0 & 0 & 0 & C_{2} & -b_{2}^{U} & a_{0}^{U+1} & 0 & 0 & 0 & \cdots & 0 \\ 0 & \ldots & 0 & 0 & 0 & 0 & 0 & c_{2} & -b_{2}^{U+1} & a_{0}^{U+2} & 0 & \cdots & \cdots & 0 \\ \vdots & \ddots & \ddots & \ddots & \ddots & & & & & \ddots & \ddots & \ddots & \ddots & \vdots \\ 0 & \ldots & 0 & 0 & 0 & 0 & 0 & 0 & 0 & 0 & c_{2} & -b_{2}^{L-4} & a_{0}^{L-3} & 0 \\ 0 & \ldots & 0 & 0 & 0 & 0 & 0 & 0 & 0 & 0 & 0 & c_{2} & -b_{2}^{L-3} & a_{0}^{L-2} \\ 0 & \ldots & 0 & 0 & 0 & 0 & 0 & 0 & 0 & 0 & 0 & 0 & c_{2} & -b_{2}^{L-2}\end{array}\right)$,

where

$$
\begin{aligned}
& C_{0}:=\left(\begin{array}{cc}
\mu_{1} & 0 \\
0 & \mu_{1}
\end{array}\right), B_{0}=\left(\begin{array}{cc}
L \lambda & 0 \\
-\mu_{2} & (L-1) \lambda+\mu_{2}
\end{array}\right), A_{0}^{k}=\left(\begin{array}{cc}
(L-k) \lambda & 0 \\
0 & (L-k-1) \lambda
\end{array}\right) \\
& B_{1}^{k}=\left(\begin{array}{cc}
(L-k-1) \lambda+\mu_{1} & 0 \\
-\mu_{2} & (L-k-2) \lambda+c_{2}
\end{array}\right), C_{1}=\left(\begin{array}{cc}
\mu_{1} & 0 \\
0 & c_{2}
\end{array}\right), A_{0}^{D-1}=\left(\begin{array}{cc}
(L-D+1) \lambda & 0 \\
0 & (L-D) \lambda
\end{array}\right), \\
& B_{1}^{D-1}=\left(\begin{array}{cc}
(L-D) \lambda+\mu_{1} & 0 \\
-\mu_{2} & (L-D-1) \lambda+c_{2}
\end{array}\right), A_{0}^{k}=\left(\begin{array}{cc}
(L-k) \lambda & 0 \\
0 & (L-k-1) \lambda
\end{array}\right), \\
& B_{2}^{k}=\left(\begin{array}{cc}
(L-k-1) \lambda+\mu_{1} & 0 \\
0 & (L-k-2) \lambda+c_{2}
\end{array}\right), C_{2}=\left(\begin{array}{ll}
0 & c_{2}
\end{array}\right), A_{0}^{U-1}=\left(\begin{array}{cc}
(L-U+1) \lambda & 0 \\
0 & (L-U) \lambda
\end{array}\right), \\
& B_{3}=\left(\begin{array}{cc}
(L-U) \lambda+\mu_{1} & -(L-U) \lambda \\
0 & (L-U-1) \lambda+c_{2}
\end{array}\right), \\
& a_{0}^{k}=A_{0}^{k} \cdot e_{2}=(L-k-1) \lambda, b_{2}^{k}=B_{2}^{k} \cdot e_{2}=(L-k-2) \lambda+c_{2}, c_{2}=C_{2} \cdot e_{2}=\mu_{1}+\mu_{2} .
\end{aligned}
$$

Proof. The statement can be proved by simple block identification at the system of balance equations taking into account defined above specifications of the subvectors. 
Theorem 3. The stationary state probabilities $\pi_{00}$ and $\pi_{k}, 0 \leq k \leq L-2$, can be calculated by

$$
\begin{aligned}
\pi_{00} & =\pi_{L-2} \prod_{i=0}^{L-2} M_{L-2-i} \\
\pi_{k} & =\pi_{L-2} \prod_{i=0}^{L-k-3} M_{L-2-i}, 0 \leq k \leq L-3 \\
\pi_{L-2} & =\left[1+\sum_{k=0}^{U-1} \prod_{i=0}^{L-k-3} M_{L-2-i} e+\sum_{k=U}^{L-3} \prod_{i=0}^{L-k-3} M_{L-2-i}\right]^{-1},
\end{aligned}
$$

where $M_{k}$ satisfies the recursive relations

$$
M_{k}= \begin{cases}C_{0} B_{0}^{-1}, & k=0 \\ C_{0}\left(B_{1}^{k-1}-M_{k-1} A_{0}^{k-1}\right)^{-1}, & 1 \leq k \leq D-1 \\ C_{1}\left(B_{1}^{D-1}-M_{D-1} A_{0}^{D-1}\right)^{-1}, & k=D \\ C_{1}\left(B_{2}^{k-1}-M_{k-1} A_{0}^{k-1}\right)^{-1}, & D+1 \leq k \leq U-1 \\ C_{2}\left(B_{3}-M_{U-1} A_{0}^{U-1}\right)^{-1}, & k=U \\ c_{2}\left(b_{2}^{k-1}-a_{0}^{k-1} M_{k-1} e_{1}\right)^{-1}, & k=U+1 \\ c_{2}\left(b_{2}^{k-1}-a_{0}^{k-1} M_{k-1}\right)^{-1}, & U+2 \leq k \leq L-2 .\end{cases}
$$

Proof. The main idea consists in deriving the recursive relations for the subvectors $\pi_{k}$ from the system of balance equations in the form

$$
\pi_{00}=\pi_{0} M_{0}, \pi_{k}=\pi_{k+1} M_{k+1},
$$

where matrices $M_{k}$ can be evaluated also by the recursive relations defined in the statement. Note that the inverse matrices which are involved into these formulas are well defined since the matrices are main diagonal dominant and hence nonsingular.

Corollary 2. The main performance measures:

- Load factor of the repair server $j=1,2$

$$
\bar{U}_{1}=\sum_{k=0}^{U-1} \pi_{k} e+\sum_{k=U}^{L-2} \pi_{k}, \bar{U}_{2}=\pi_{00} e_{1}+\sum_{k=0}^{U-1} \pi_{k} e_{1}+\sum_{k=U}^{L-2} \pi_{k}
$$

- Mean number of busy servers $\bar{C}=\sum_{j=1}^{2} \bar{U}_{j}$;

- Mean number of failed machines in the buffer

$$
\bar{Q}=\sum_{k=0}^{U-1} k \pi_{k} e+\sum_{k=U}^{L-2} k \pi_{k}
$$


- Mean number of failed machines in the system $\bar{N}=\bar{C}+\bar{Q}$;

- Mean waiting and sojourn time of the failed machine

$$
\bar{W}=\frac{\bar{Q}}{\lambda(L-\bar{N})}, \bar{T}=\frac{\bar{N}}{\lambda(L-\bar{N})} ;
$$

$-\mathbb{P}[$ Machine $n$ is failed $]=\frac{\lambda \bar{W}}{\lambda \bar{W}+1}$;

- The mean cost per unit of time

$$
g(U, D)=c_{0} \bar{Q}+\sum_{j=1}^{2} c_{j} \bar{U}_{j}+c_{01} \lambda(L-U) \pi_{U-1} e_{0}+c_{10} \mu_{2} \sum_{k=0}^{D-1} \pi_{k} e_{1} .
$$

\section{$5 \quad$ Numerical Examples}

In this section we discuss some interesting observations about the properties of the optimal control policy $f=\left(f_{0}, f_{1}, f_{2}\right)$. To evaluate optimal policies we apply the Howard iteration algorithm [3] and formulas obtained in previous section.

Table 1. Component $f_{0}$ of the (a) optimal control policy (b) hysteretic control policy

(a)

\begin{tabular}{|c||c|c|c|c|c|c|c|}
\hline \multicolumn{1}{|c||}{ System State $x$} & \multicolumn{6}{c|}{ Queue Length $q(x)$} \\
\hline$\left(d_{1}, d_{2}\right)$ & 0 & 1 & 2 & 3 & 4 & $\ldots$ & 15 \\
\hline \hline$(0,0)$ & 1 & 1 & 1 & 1 & 1 & $\ldots$ & 1 \\
\hline$(0,1)$ & 0 & 0 & 1 & 1 & 1 & $\ldots$ & 1 \\
\hline$(1,0)$ & 0 & 0 & 0 & 2 & 2 & $\ldots$ & 2 \\
\hline$(1,1)$ & 0 & 0 & 0 & 0 & 0 & $\ldots$ & 0 \\
\hline
\end{tabular}

(b)

\begin{tabular}{|c||c|c|c|c|c|c|c|}
\hline \multicolumn{1}{|c||}{ System State $x$} & \multicolumn{7}{c|}{ Queue Length $q(x)$} \\
\hline$\left(d_{1}, d_{2}\right)$ & 0 & 1 & 2 & 3 & 5 & $\ldots$ & 15 \\
\hline \hline$(0,0)$ & 1 & 1 & 1 & 1 & 1 & $\ldots$ & 1 \\
\hline$(0,1)$ & 1 & 1 & 1 & 1 & 1 & $\ldots$ & 1 \\
\hline$(1,0)$ & 0 & 0 & 0 & 2 & 2 & $\ldots$ & 2 \\
\hline$(1,1)$ & 0 & 0 & 0 & 0 & 0 & $\ldots$ & 0 \\
\hline
\end{tabular}

Table 2. Component $f_{1}$ of the (a) optimal control policy (b) hysteretic control policy

(a)

\begin{tabular}{|c||c|c|c|c|c|c|c|}
\hline \multicolumn{1}{|c||}{ System State $x$} & \multicolumn{6}{|c|}{ Queue Length $q(x)$} \\
\hline$\left(d_{1}, d_{2}\right)$ & 1 & 2 & 3 & 4 & 5 & $\ldots$ & 15 \\
\hline \hline$(1,0)$ & 1 & 1 & 1 & 1 & 1 & $\ldots$ & 1 \\
\hline$(1,1)$ & 0 & 0 & 1 & 1 & 1 & $\ldots$ & 1 \\
\hline
\end{tabular}

\begin{tabular}{|c||c|c|c|c|c|c|c|}
\hline System State $x$ & \multicolumn{6}{|c|}{ Queue Length $q(x)$} \\
\hline$\left(d_{1}, d_{2}\right)$ & 1 & 2 & 3 & 4 & 5 & $\ldots$ & 15 \\
\hline \hline$(1,0)$ & 1 & 1 & 1 & 1 & 1 & $\ldots$ & 1 \\
\hline$(1,1)$ & 1 & 1 & 1 & 1 & 1 & $\ldots$ & 1 \\
\hline
\end{tabular}

Table 3. Component $f_{2}$ of the (a) optimal control policy (b) hysteretic control policy

(a)

\begin{tabular}{|c||c|c|c|c|c|c|c|}
\hline System State $x$ & \multicolumn{6}{|c|}{ Queue Length $q(x)$} \\
\hline$\left(d_{1}, d_{2}\right)$ & 1 & 2 & 3 & 4 & 5 & $\ldots$ & 15 \\
\hline \hline$(0,1)$ & 2 & 2 & 2 & 2 & 2 & $\ldots$ & 2 \\
\hline$(1,1)$ & 2 & 2 & 2 & 2 & 2 & $\ldots$ & 2 \\
\hline
\end{tabular}

(b)

\begin{tabular}{|c||c|c|c|c|c|c|c|}
\hline System State $x$ & \multicolumn{6}{|c|}{ Queue Length $q(x)$} \\
\hline$\left(d_{1}, d_{2}\right)$ & 1 & 2 & 3 & 4 & 5 & $\ldots$ & 15 \\
\hline \hline$(0,1)$ & 2 & 2 & 2 & 2 & 2 & $\ldots$ & 2 \\
\hline$(1,1)$ & 2 & 2 & 2 & 2 & 2 & $\ldots$ & 2 \\
\hline
\end{tabular}


(a)

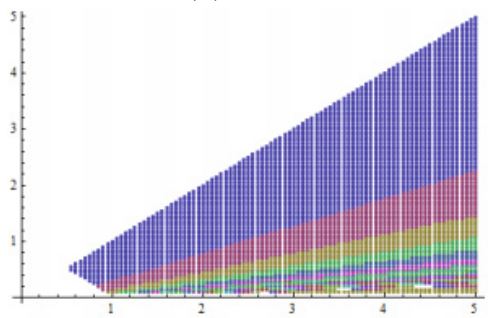

(b)

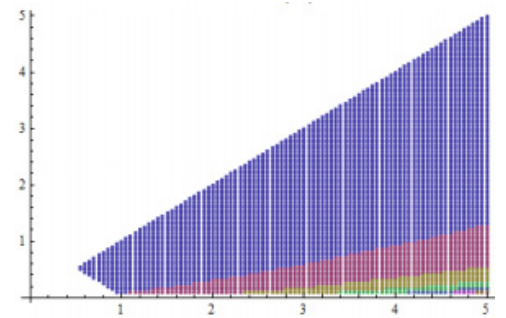

Fig. 2. The regions of optimality for threshold $U$ in (a) infinite population (b) finite population $L=10$ model

If the switching $\operatorname{costs} c_{01}$ and $c_{10}$ are set to be equal to 0 , than, as expected, the optimal control policy $f$ is of threshold type, i.e. is defined through a single threshold level, $U=D>0$, like at a similar system with infinite population. Due to this policy the second server with a higher cost per service period $\frac{c_{2}}{\mu_{2}}>\frac{c_{1}}{\mu_{1}}$ must be used in state $x=(q, 1,0)$ whenever the number of failed machines in the buffer exceeds the threshold level $U, q(x)>U$.

If the switching costs are differs from 0 for the most values of system parameters $\lambda, \mu_{1}$ and $\mu_{2}$ the optimal policy belongs to the hysteretic policy with two threshold levels, $(U, D)$, discussed at the beginning of the previous section. This policy is optimal in infinite population case as well. But surprisingly this policy for the closed system is not optimal everywhere, i.e. for some values of system parameters, e.g. if $c_{01}$ is very large comparing to other costs, one more threshold level appears for the activation of the first server in state $x=(q, 0,1)$.

Tables 1-3 illustrate the components $f_{j}, j=0,1,2$, of the optimal control policy (OCP) and the hysteretic control policy (HCP), $(4,1)$, for the following values,

$$
L=17, \lambda=1, \mu_{1}=5, \mu_{2}=1, c_{0}=c_{1}=c_{2}=1, c_{01}=50, c_{10}=5 .
$$

The gain function for these policies are equal to

$$
g_{\mathrm{OCP}}=11.0125, g_{\mathrm{HCP}}=11.0524 .
$$

As we can see, the difference is not sufficient. In other numerical examples the observable difference in performance was not more than $0.5 \%$, so the hysteretic policy can be treated as a quasi-optimal one.

Another observation concerns the optimal threshold policy with threshold level $U$ if $c_{0}=c_{1}=c_{2}=1$ and $c_{01}=c_{10}=0$. The areas of optimality for threshold level depending on ratios $r_{1}=\frac{\mu_{1}}{\lambda}$ and $r_{2}=\frac{\mu_{2}}{\lambda}$ for infinite and finite population models are shown in Figure 2. The larger upper region stands for the case $U=1$, below is shown the region for $U=2$ and so on. We observe that the slope in the finite case is flatter. In finite population case the faster server must be more than four time faster as the slower one to get non-trivial solution $U>1$, otherwise the optimal threshold policy will coincide with the fastest free 
server discipline $U=1$, where the fastest available server must be used each time there is a waiting machine in the buffer.

\section{Conclusion}

In this paper we have studied a controllable machine repairman model with heterogeneous repair servers. For the model without switching costs the optimal control policy has a threshold structure. We expect that this fact can be rigorously proved using event-based dynamic programming approach to prove the monotonicity properties of the value function in the same way as it was done for infinite population models. In general case with switching costs the optimal control policy can be more complicated as a known hysteretic policy but the difference in performance between the policies is negligible.

\section{References}

1. Artalejo, J.R., Economou, A.: Markovian controllable queueing systems with hysteretic policies: Busy period and waiting time analysis. Method. and Comp. in App. Prob. 7, 353-378 (2005)

2. Falin, G.I.: A multiserver retrial queue with a finite number of sources of primary calls. Math. and Comp. Mod. 20, 33-49 (1999)

3. Howard, R.: Dynamic Programming and Markov Processes. Wiley Series, New York (1960)

4. Mitrani, I.: Managing performance and power consumption in a server farm. Ann. Oper. Res. 202, 121-134 (2013)

5. Nobel, R., Tijms, H.C.: Optimal control of a queueing system with heterogeneous servers and set-up costs. IEEE Transactions on Automation Control 45(4), 780-784 (2000)

6. Le Ny, L.M., Tuffin, B.: A simple analysis of heterogeneous multi-server threshold queues with hysteresis. In: Proceedings of Applied Telecommunication Symposium (ATS) (2002)

7. Puterman, M.L.: Markov Decision Process: Discrete Stochastic Dynamic Programming. John Wiley\&Sons, New-York (1994)

8. Tijms, H.C.: Stochastic Models. An Algorithmic Approach. John Wiley\&Sons, New-York (1994)

9. Roszik, J., Sztrik, J.: Performance analysis of finite-source retrial queues with nonreliable heterogeneous servers. J. of Math. Sciences 146, 6033-6038 (2007)

10. Rykov, V., Efrosinin, D.: On the slow server problem. Autom. and Rem. Control 70(12), 2013-2023 (2009) 